\title{
Achados ultrassonográficos da endometriose: principais apresentações e aspectos atípicos - ensaio iconográfico
}

\section{Ultrasonographic findings of endometriosis: main presentations and atypical aspects - iconographic test}

\author{
Renan Mesquita Soares ${ }^{1}$. Jesus Irajacy Fernandes da Costa² .
}

1 Médico residente de Radiologia e Diagnóstico por Imagem, Hospital Universitário Walter Cantídio (HUWC), Universidade Federal do Ceará (UFC), Fortaleza, Ceará, Brasil. 2 Mestre em Medicina, Professor Assistente, Departamento de Medicina Clínica, Universidade Federal do Ceará (UFC), Fortaleza, Ceará, Brasil.

\section{RESUMO}

A endometriose é uma importante enfermidade feminina, muitas vezes causando dor pélvica crônica e infertilidade, pois tal condição pode se apresentar como cistos hemorrágicos ovarianos, nódulos e aderências peritoneais. Neste contexto, os exames de imagem tornam-se fundamentais para o diagnóstico, sendo que as técnicas de imagem mais utilizadas são a ultrassonografia transvaginal (USTV) após preparo intestinal e a ressonância magnética (RM). Apesar da RM ser considerada o melhor exame de imagem para o diagnóstico, a USTV apresenta papel fundamental para a detecção de lesões em locais como torus uterino, ovários e fundo de saco de Douglas. Através de aparelho de ultrassonografia padrão, e após o preparo adequado do paciente, o presente estudo tem como objetivo apresentar alguns achados da ultrassonografia transvaginal de apresentações comuns e incomuns desta patologia.

Palavras-chave: Endometriose. Ultrassonografia. Radiologia.

\section{ABSTRACT}

Endometriosis is an important women's disease, often causing chronic pelvic pain and infertility. This condition may present as ovarian hemorrhagic cysts, nodules and peritoneal adhesions. In this context, the imaging studies become fundamental for the diagnosis. The most commonly used imaging techniques are transvaginal sonography (TVS) after bowel preparation and magnetic resonance imaging (MRI). Although MRI is considered the best test image for diagnosis, TVS has an excellent accuracy for the detection of lesions in diferente places such as uterine torus, ovaries and Douglas pouch. The aim of the present study was to present some findings of transvaginal ultrasonography of common and uncommon presentations of this pathology by means of a standard ultrasound device and after adequate preparation of the patient.

Keywords: Endometriosis. Ultrasonography. Radiology.

Autor correspondente: Renan Mesquita Soares, Rua Thompson Bulcão, 421, Engenheiro Luciano Cavalcante, Fortaleza, Ceará. CEP: $60810-460$. Telefone: +55 85 98878-9876. E-mail: renanmesquita2@gmail.com

Conflito de interesses: Não há qualquer conflito de interesses por parte de qualquer um dos autores.

Recebido em: 08 Jan 2018; Revisado em: 10 Mar 2018; Aceito em: 25 Abr 2018. 


\section{INTRODUÇÃO}

A endometriose é uma patologia ginecológica crônica caracterizada por tecido endometrial fora da cavidade uterina, manifestada, principalmente, por implantes peritoneais ou cistos hemorrágicos ovarianos. Se a lesão peritoneal estiver infiltrada além de $5 \mathrm{~mm}$ da superfície peritoneal, considera-se como endometriose profunda. ${ }^{1,2}$ A endometriose afeta, aproximadamente, $10 \%$ a $15 \%$ das mulheres em idade reprodutiva e pode ser encontrada em cerca de $70 \%$ das mulheres com dor pélvica crônica. ${ }^{3}$

Vários mecanismos patogênicos têm sido propostos, porém, atualmente aceita-se como principais etiologias a menstruação retrógrada, implantação de células tronco endometriais, anormalidades do remanescente Mülleriano, e metaplasia celômica. ${ }^{4}$ Há estudos demonstrando mediadores inflamatórios envolvidos, associada a fatores epigenéticos e herança poligênica-multifatorial. ${ }^{4}$

Os principais sintomas são dor e infertilidade, resultantes de insultos inflamatórios crônicos. Ainda, ansiedade, dor lombar, fadiga, disúria e hematúria são as manifestações clínicas mais comuns e relevantes. ${ }^{5}$

A ultrassonografia transvaginal (USTV) realizada após preparo intestinal deve ser o exame de imagem de primeira linha quando se suspeita da presença de endometriose. Este método é mais preciso que o US transretal, e produz melhores resultados do que a ressonância magnética (RM) para a avaliação de implantes profundos (especialmente os de pequeno volume), de lesões ovarianas e vesicais. ${ }^{6,7}$

\section{PROCEDIMENTO E PREPARAÇÃO DO PACIENTE}

A USTV é realizada em aparelho de ultrassom (US), Sonosite ICT Toshiba, com transdutores de frequência entre 5 e $9 \mathrm{MHz}$ para estudo endocavitário e de 2 a $4 \mathrm{MHz}$ para avaliação abdominal, após preparo intestinal - este preparo é usado para eliminar conteúdo fecal e gás no cólon sigmoide. Ele inclui um laxante suave administrado em duas doses orais (às $8 \mathrm{~h}$ e às $14 \mathrm{~h}$ ) no dia anterior ao exame agendado, uma dieta de baixo resíduo por 24 horas antes do exame e um enema (120 $\mathrm{mL}$ de difosfato de sódio) administrado cerca de uma hora antes do procedimento.

A duração do exame é variável a depender da complexidade das lesões e da experiência do examinador, com média de 30 minutos. O algoritmo de avaliação de imagem inicia-se desde a cavidade abdominal superior, incluindo cúpulas diafragmáticas, espaços hepatorrenal e esplenorrenal e superfície hepática, estendendo-se ao estudo de alças intestinais utilizandose transdutor de alta resolução, à avaliação da bexiga, e finalizando com o estudo transvaginal que inclui o estudo do útero, dos ovários, do peritônio pélvico que cobre a bexiga, dos fundos de saco, dos ligamentos uterossacros, do reto e do cólon sigmoide. A parede da bexiga, inserção dos ligamentos redondos, septo retovaginal e fórnice vaginal posterior são igualmente avaliados. O cólon sigmoide é rastreado da borda anal à transição sigmoide-cólon descendente.
$\mathrm{Na}$ avaliação de implantes em alças intestinais, é imprescindível informar qual a porção da circunferência que é afetada, estimativa da área circunferencial acometida e o grau de penetração através da serosa. É importante realizar a ultrassonografia em conjunto com a palpação abdominal para a pesquisa de aderências.

\section{ACHADOS ULTRASSONOGRÁFICOS DA ENDOMETRIOSE}

A USTV não é o método de escolha na detecção de implantes peritoniais. ${ }^{8}$ Embora seja incapaz de alcançar determinadas topografias, ele é importante devido à avaliação dinâmica. A USTV é o melhor exame para a detecção de endometriomas, pois consegue mostrar algumas características fundamentais para o seu diagnóstico. Os endometriomas encontram-se entre as lesões mais comuns desta enfermidade e se apresentam da seguinte forma:

1) lesões homogêneas focais, de contornos regulares e limites definidos, ocupando a subtotalidade do estroma ovariano, exibindo pequenos ecos em seu interior, por vezes móveis, além de focos puntiformes hiperecóicos periféricos; aspecto ultrassonográfico que lembra a ecotextura sólida e homogênea do testículo (Figura 1).

2) são tipicamente uniloculares, porém, podem ser multiloculares, contendo finas ou grosseiras septações em permeio, fato que pode ser justificado por diferentes momentos da degradação sanguínea (Figura 2A).

3) ao estudo Doppler fluxométrico não se observa vascularização interna (Figuras $2 \mathrm{~A}$ e $2 \mathrm{~B}$ ).

Ambos os ovários podem estar fixos devido às aderências formadas, algumas causadas por coleções na cavidade pélvica. Quando os ovários se aproximam da linha média e são fixos um ao outro, chamamos de sinal do beijo ovariano ("kissing ovaries") (Figuras 3 e 4).

Os implantes endometrióticos podem se apresentar como revestimento hipoecoico recobrindo a superfície peritoneal, chamados de lesões em manto. Tais apresentações podem ser confundidas com varizes pélvicas, achado bastante comum em mulheres em idade fértil. Por este motivo, é fundamental o estudo Doppler fluxométrico para o diagnóstico diferencial (Figura 5).

Implantes endometrióticos focais apresentam-se como nódulos sólidos, hipoecoicos, finamente heterogêneos, de contornos lobulados ou microlobulados e limites bem definidos, localizados nas mais diversas topografias.

A endometriose intestinal é uma forma bastante comum de apresentação da endometriose, e mostra-se como nódulos hipoecoicos subserosos aderidos à serosa (Figura 6).

Algumas vezes os implantes endometrióticos apresentam topografias não habituais como os descritos, podendo 
alcançar locais como a cicatriz umbilical (Figura 7) e a parede abdominal (Figura 8). Porém, é necessário excelente conhecimento anatômico para não haver confusão entre os achados (Figura 9).
Focos endometrióticos também podem estar localizados no miométrio, por vezes simulando principalmente miomas uterinos (Figura 10). As principais queixas relatadas pelas pacientes nesses casos são dispareunia e dismenorreia.

Figura 1. Lesão endometriótica clássica ovariana.

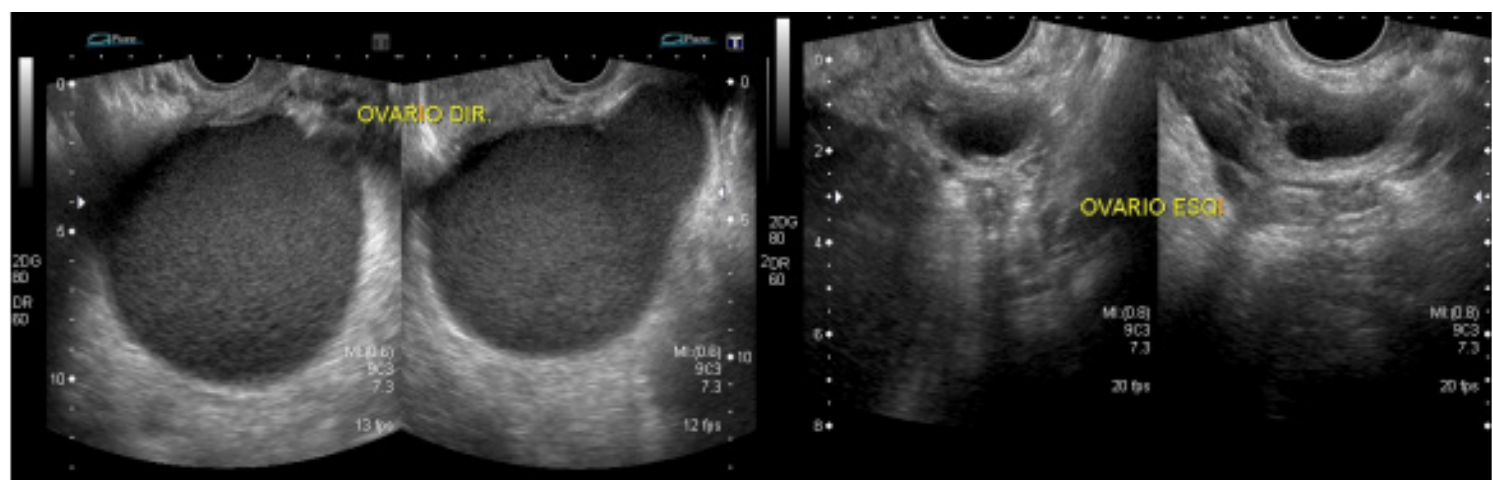

Nota: Nota-se o ovário direito de dimensões bastante aumentadas em relação ao contralateral, exibindo volumosa formação cística, de paredes finas e limites bem definidos, evidenciando em seu conteúdo finos ecos em suspensão e diminutos focos hiperecoicos periféricos, achados que são sugestivos de endometrioma. Tal aspecto lembra o aspecto ultrassonográfico sólido e homogêneo de um testículo. Ovário esquerdo de aspecto habitual, exibindo pequena formação cística simples que corresponde a folículo de natureza funcional

Figura 2. Lesão endometriótica ovariana de aspecto atípico, com estudo dopplerfluxométrico.

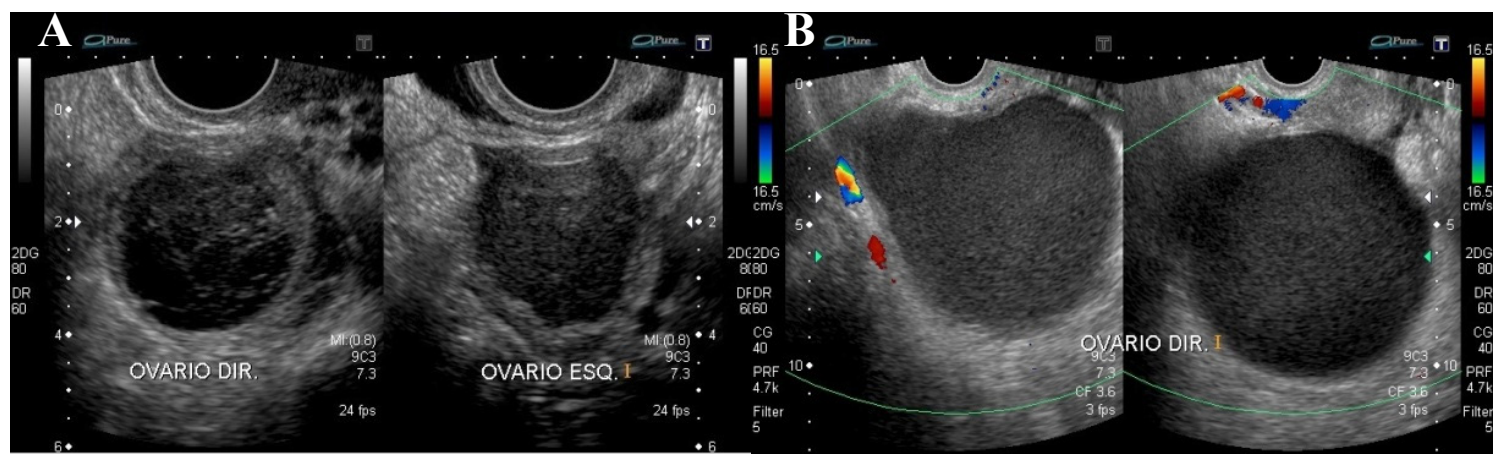

Nota: Formação cística (imagem à direita) com características de endometrioma em ovário esquerdo, conforme descrito anteriormente; observa-se outra formação cística em ovário direito, de paredes finas, contornos regulares e limites definidos, exibindo finas e grosseiras septações em permeio (imagem à direita), que foi confirmado como outro endometrioma após laparoscopia. O aspecto ultrassonográfico não habitual é justificado por fases diferentes da degradação sanguínea.

Figura 3. Aderências periovarianas.

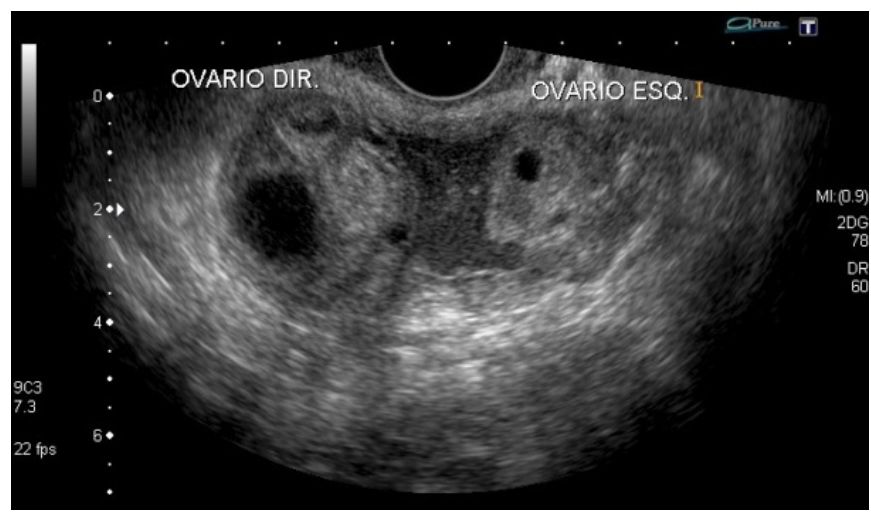

Nota: Observam-se os ovários próximos, aparentemente unidos por coleção finamente heterogênea, cujo aspecto de seu conteúdo assemelhase a um endometrioma, mostrando sua natureza hemorrágica. Durante a realização do exame não houve mobilização de ambos os ovários.
Figura 4. Coleção periovariana.

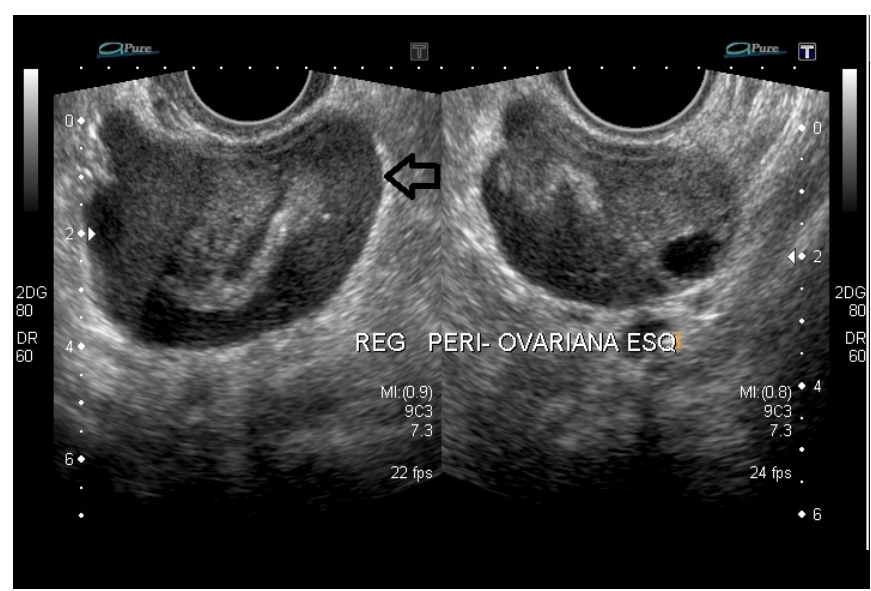

Nota: Coleção finamente heterogênea (seta preta), com aspecto semelhante a endometrioma envolvendo o ovário esquerdo. 
Figura 5. Diagnóstico diferencial de endometriose pélvica, com estudo dopplerfluxométrico.

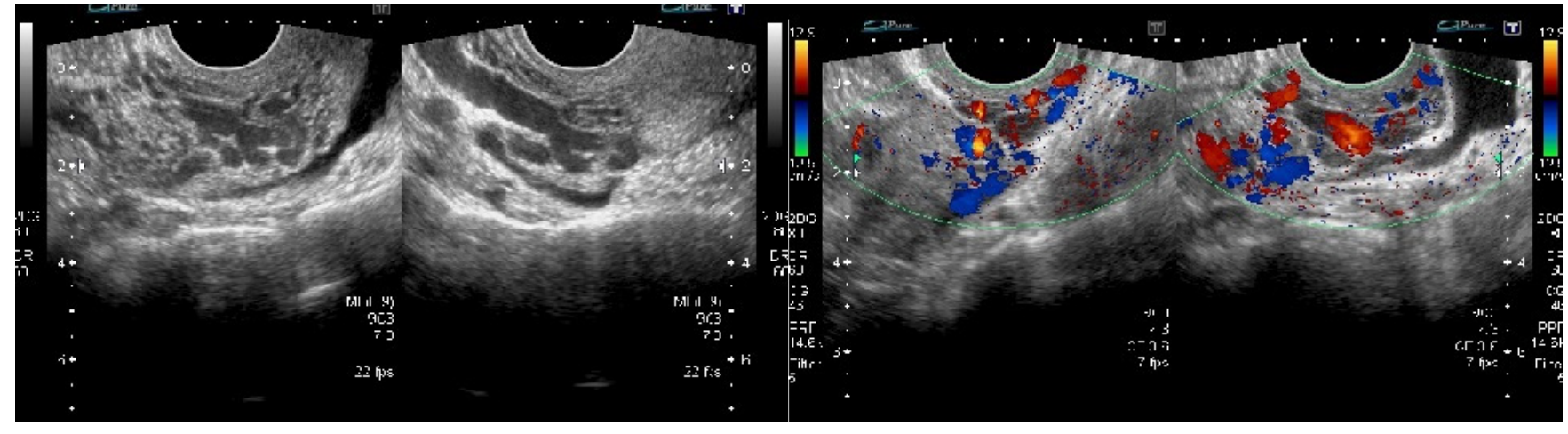

Nota: Ultrassonografia transvaginal para pesquisa de endometriose evidenciou estruturas tubulares, serpiginosas, localizadas na cavidade pélvica próximas ao istmo uterino, recobrindo a superfície peritoneal da cavidade pélvica. Ao estudo Doppler fluxométrico colorido, notou-se que são estruturas vasculares. Sua semelhança ocorre pelo baixo fluxo sanguíneo, por vezes com momentos de estase sanguínea.

Figura 6. Endometriose intestinal.

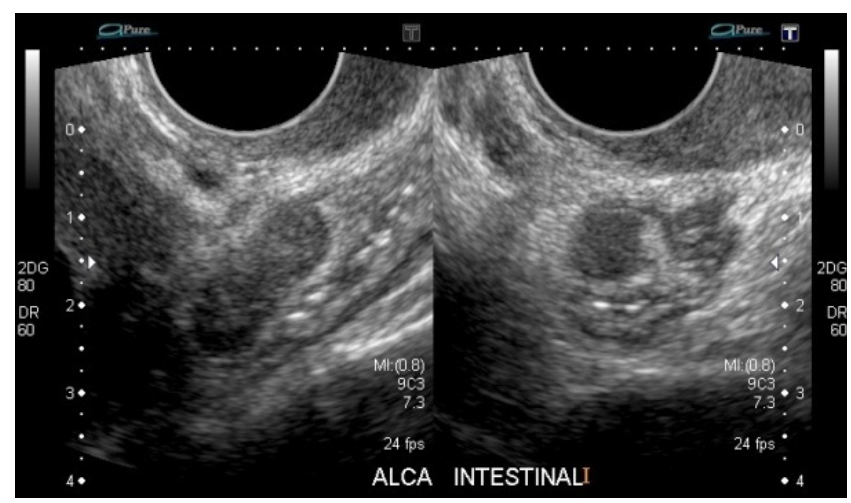

Nota: Nódulo sólido, hipoecoico, de contornos lobulados e limites definidos, localizado na região subserosa da alça intestinal da fossa ilíaca esquerda, consistente com foco endometriótico.

Figura 7. Encometriose em cicatriz umbilical.

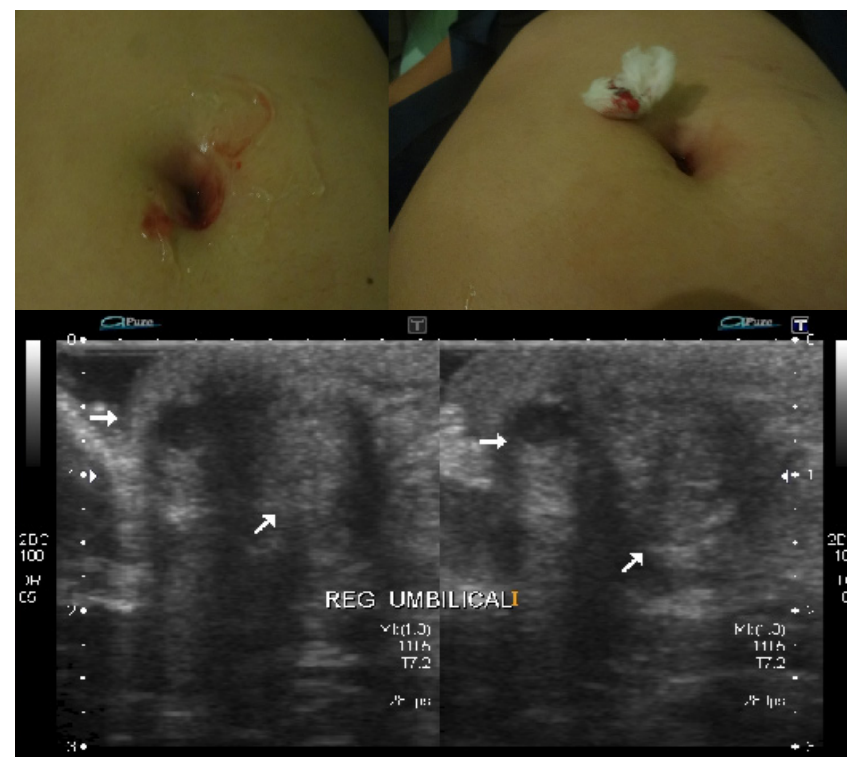

Nota: Paciente com 26 anos apresenta queixa de sangramento intermitente através da cicatriz umbilical há 8 meses. Ultrassonografia da parede abdominal evidenciou nódulos sólidos hipoecoicos, parcialmente definidos, localizados no plano subcutâneo da cicatriz umbilical.
Figura 8. Endometriose em parede abdominal.

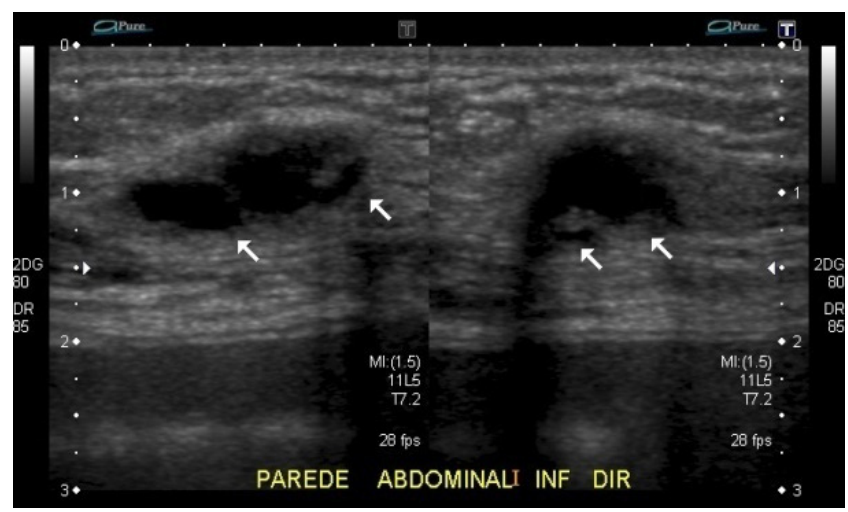

Nota: Paciente com exame anterior evidenciando endometrioma em ambos os ovários, relatou dor em fossa ilíaca direita. Exame ultrassonográfico da parede abdominal mostrou nódulo sólido hipoecoico, de contornos lobulados e limites definidos, em topografia do subcutâneo, consistente com foco endometriótico.

Figura 9. Falso positivo ultrassonográfico.

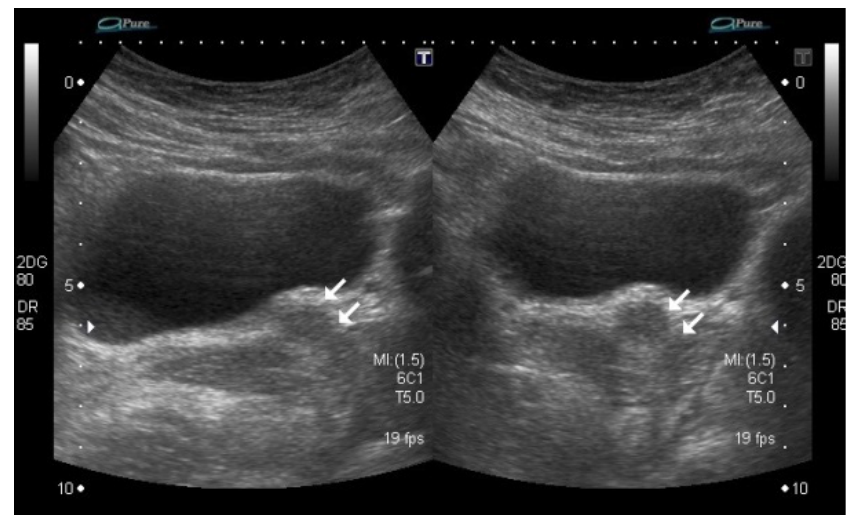

Nota: Paciente de 32 anos com dispareunia de profundidade há um ano. Realizou ultrassonografia que foi sugestiva de foco endometriótico em terço proximal da uretra. Realizou nova ultrassonografia que identificou em provável topografia descrita apenas a uretra de aspecto habitual. Realizada cistoscopia após duas semanas com resultados normais. 
Figura 10. Endometriose uterina.

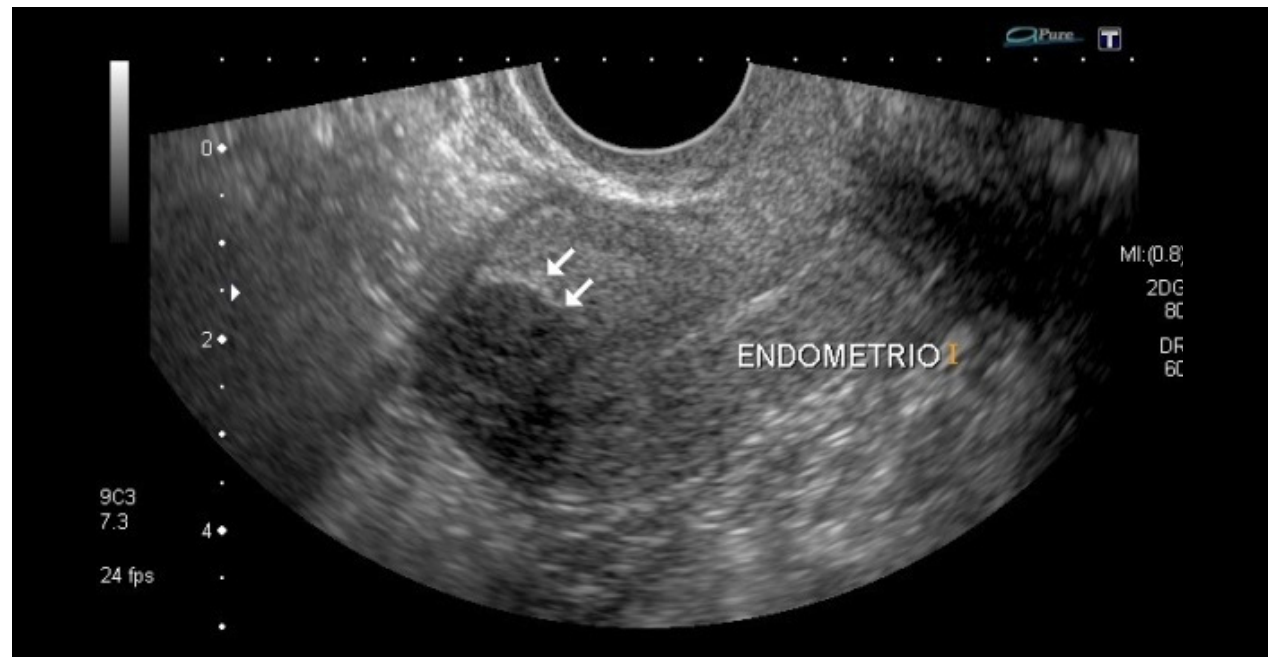

Nota: Nódulo sólido, hipoeoico, bem definido, de ecotextura semelhante a um endometrioma localizado na região fúndica uterina, estendendo-se desde a serosa até a cavidade endometrial.

\section{CONCLUSÃO}

A USTV é o primeiro exame de escolha para o diagnóstico de endometriose por ser rápido, barato e de fácil acesso.

\section{REFERÊNCIAS}

1 Bulun SE. Endometriosis. N Engl J Med. 2009;360(3):268-79.

2 Parasar P, Ozcan P, Terry KL. Endometriosis: epidemiology, diagnosis and clinical management. Curr Obstet Gynecol Rep. 2017;6(1):34-41.

3 The American College of Obstetricians and Gynecologists. Practice Bulletin $\mathrm{n}^{\circ}$ 14: Management of endometriosis. Obstet Gynecol. 2010;114(1):223-36.

4 Vercellini, P, Viganò P, Somigliana E, Fedele L. Endometriosis: pathogenesis and treatment. Nat Rev Endocrinol. 2014;10(5):261-75.

5 Soliman AM, Coyne KS, Zaiser E, Castelli-Haley J, Fuldeore MJ. The burden of endometriosis symptoms on health-related quality of life in women in the United States: a cross-sectional study. J Psychosom Obstet Gynaecol. 2017;38(4):238-48.
Apresentamos casos de nossa experiência para demonstrar os principais achados, alguns incomuns; outros merecendo diagnósticos diferenciais.

6 Gonçalves MO, Dias JA Junior, Podgaec S, Abrão MS. Transvaginal ultrasound for diagnosis of deeply infiltrating endometriosis. Int $\mathrm{J}$ Gynaecol Obstet. 2008;104(2):156-60.

7 Chamié LP, Pereira RM, Zanatta A, Serafini PC. Transvaginal US after bowel preparation for deeply infiltrating endometriosis: protocol, imaging appearances, and laparoscopic correlation. RadioGraphics. 2010;30(5):1235-49.

8 Ros C, Martínez-Serrano MJ, Rius M, Abrao MS, Munrós J, Martínez-Zamora MA, et al. Bowel preparation improves the accuracy of the transvaginal ultrasound in the diagnosis of rectosigmoid deep infiltrating endometriosis: a prospective study. J Minim Invasive Gynecol. 2017;24(7):1145-51.

\section{Como citar:}

Soares RM, Costa JI. Achados ultrassonográficos da endometriose: principais apresentações e aspectos atípicos - ensaio iconográfico. Rev Med UFC. 2018 out-dez;58(4):52-56. 